\title{
Bilateral Papilledema as the First Sign of Ventriculoperitoneal Shunt Dysfunction $^{\S}$
}

\author{
Juan Carlos Serna-Ojeda*, Montserrat Aguirre-Mireles and Mayra Fabiola Camargo-Suarez
}

Instituto de Oftalmologia “Conde de Valenciana”. Mexico City, DF, Mexico

\begin{abstract}
A 12 year-old girl patient with a history of ventriculoperitoneal shunt placement at the age of 6 months presented with progressive bilateral decrease in visual acuity. Ophthalmologic examination was consistent with bilateral papilledema. No other systemic manifestations of increased intracranial pressure were evident and laboratory work-up excluded other inflammatory or infectious processes. We present here an interesting case of a patient with ophthalmologic manifestations as the initial finding of ventriculoperitoneal shunt dysfunction.
\end{abstract}

Keywords: Bilateral papilledema, papilledema, ventriculoperitoneal shunt.

\section{INTRODUCTION}

The usual clinical presentation of ventriculoperitoneal valve dysfunction in children with full cranial development includes headache, vomiting, nausea and lethargy; bilateral papilledema is present due to increased intracranial pressure. The probability of shunt dysfunction is high, reported in up to $81 \%$ of cases, 12 years after surgical follow-up [1], and can be due to mechanical complications such as obstruction, disconnection, catheter migration or equipment failure as well as infectious processes. We herein report a case of ventriculoperitoneal shunt dysfunction in a patient that only presented ophthalmological abnormalities.

\section{CASE REPORT}

A 12 year-old female patient with a history of ventriculoperitoneal shunt placement at the age of 6 months due to hydrocephaly, was evaluated for bilateral decrease in visual acuity of 4 months of evolution. She had previously been administered oral prednisone because of a presumptive diagnosis of bilateral optic neuritis at another institution.

On examination, her right eye visual acuity was limited to hand movements and the left eye was $2 / 200$. Torch light examination revealed pupillary mydriasis with equal pupillary size, and bilateral abnormal light and consensual reflexes. No cranial nerve palsies were evident in the ocular movements exam.

Both eye fundi had generalized paleness $(4+)$, elevated and blurred borders of both optic discs; from their emergence there was sheathing of the central blood vessels, were tortuous, with gliosis and lacking of a venous pulse. The macula had scattered hard exudates, Paton lines, retinal folds and no lesions at the peripheral retina (Fig. 1).

\footnotetext{
*Address correspondence to this author at the Instituto de Oftalmologia "Conde de Valenciana", Chimalpopoca 14, 06800 Mexico City, DF, Mexico; Tel:+52 5554421700; Fax: +52 5554421700;

E-mail: juanc.sernao@gmail.com

${ }^{\S}$ Partially presented at the XXXII Mexican Congress of Ophthalmology. Cancun, Mexico. June 2014.
}

Retinal fluorescein angiography obtained in another institution, revealed early hyperfluorescence due to papillary leakage.

Goldmann visual fields revealed a concentric decrease of the peripheral visual fields with a right eye 25-30 degree remaining central field on IV/E3 stimulation, and a 20-30 central field in the left eye, also on IV/E3 stimulation (Fig. 2).

Brain magnetic resonance imaging showed the ventricular system in the midline mild bilateral ventricular dilation, an increase in supratentorial volume with no associated subependymal edema, slightly decreased overall encephalic volume for the patient's age and no causes of intracranial hypertension like tumors or hematomas (Fig. 3).

The patient never developed other systemic manifestations of increased intracranial pressure such as headache, vomiting, nausea or lethargy other than the reported progressive visual deterioration and late-stage pupillary abnormalities with bilateral papilledema. An extensive laboratory work-up was conducted that excluded other inflammatory or infectious processes. She was then referred for pediatric neurologic evaluation with the diagnosis of ventriculoperitoneal shunt dysfunction with ophthalmologic manifestations, with subsequent confirmation of the diagnosis by the referral institution.

\section{DISCUSSION AND CONCLUSION}

We have presented the case report of a patient with ophthalmologic manifestations of decreased visual acuity and chronic, bilateral papilledema as the initial finding of ventriculoperitoneal shunt dysfunction. Although symptoms of increased intracranial pressure such as headache, nausea, lethargy or diplopia are expected, these may be absent if the intracranial pressure increases slowly; [2] the development of papilledema requires a critical time period of pressure elevation.

The signs detected by imaging studies in these cases include ventriculomegaly, flattening of the sulci and changes 

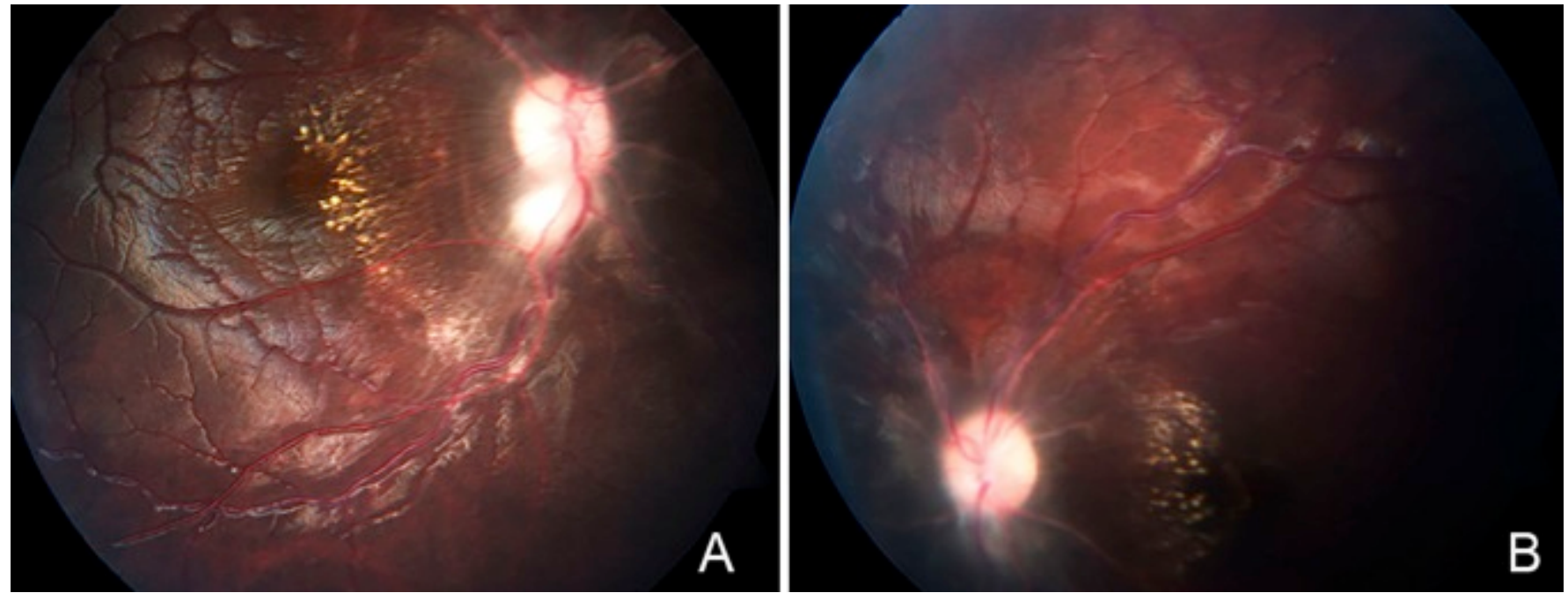

Fig. (1). A. Clinical image of the right fundus showing scattered macular exudates and Paton lines. B. Clinical image of the left fundus showing a pale disc and blurred borders.
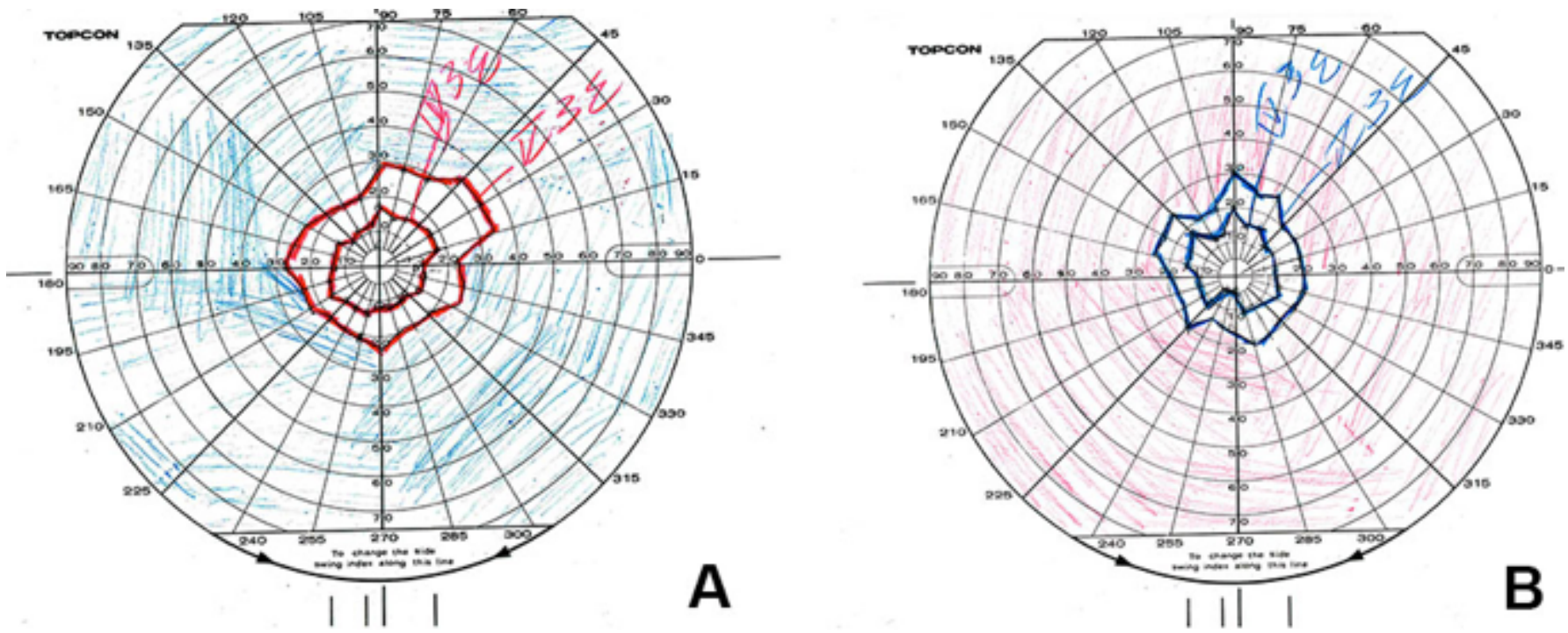

Fig. (2). Goldmann visual fields. Concentric decrease of the peripheral visual fields is shown from right eye (A) and left eye (B).

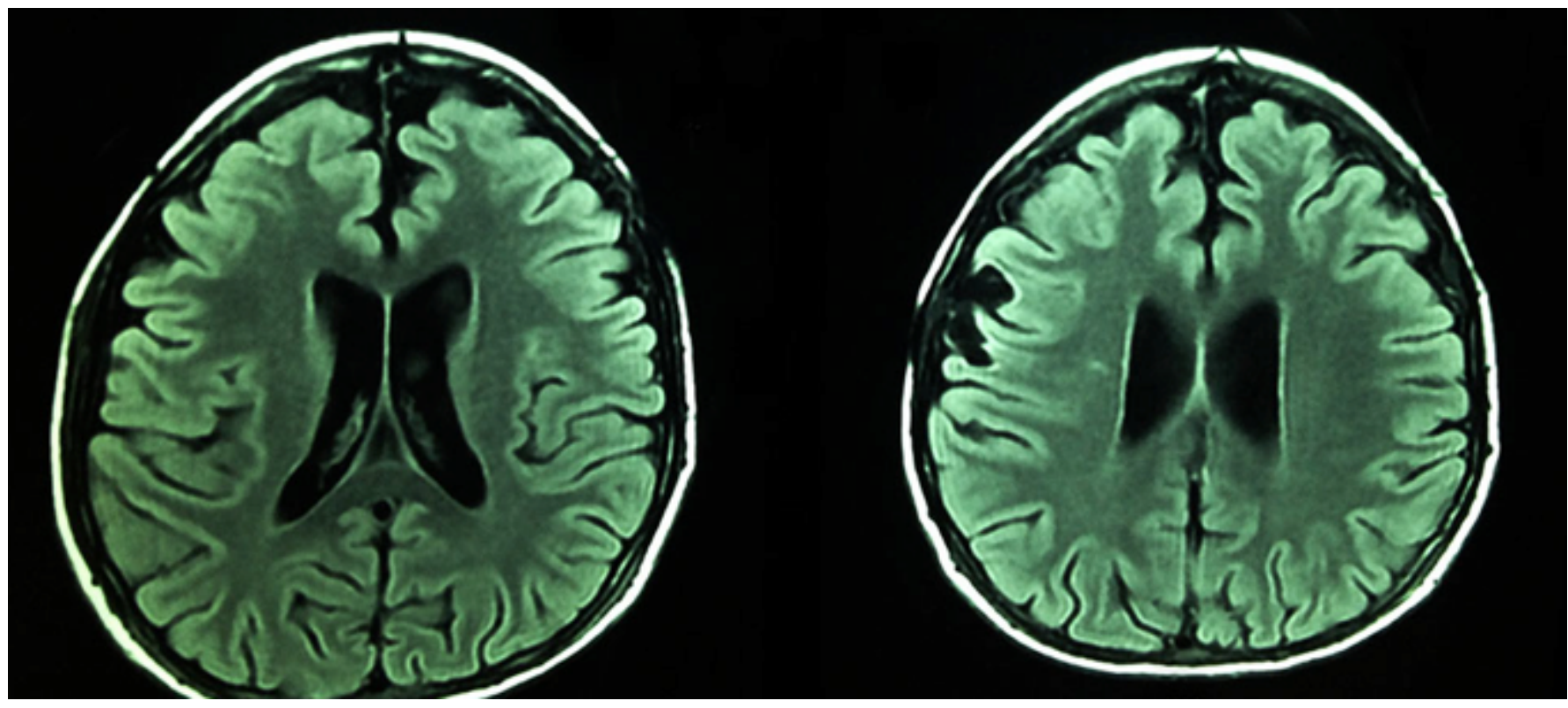

Fig. (3). Brain magnetic resonance imaging, axial views. 
in the periventricular white matter's signal intensity; [2] these findings however, are not always evident as in our patient's case in whom only the brain changes due to the initial hydrocephalus were apparent.

In older children, shunt dysfunction may develop without associated clinical manifestations including the loss of neither visual acuity nor obvious abnormalities on neuroimaging; hence, a thorough ophthalmological examination is essential [3]. It is crucial to remember that although papilledema is not a sensitive sign, it is still considered a pivotal ophthalmological finding suggesting shunt dysfunction [4].

If the ophthalmological manifestations, such as decreased visual acuity among other signs, are missed or misinterpreted in presence of a normal overall examination, severe and permanent loss of vision may ensue $[5,6]$.

\section{CONFLICT OF INTEREST}

The authors confirm that this article content has no conflict of interest.

\section{ACKNOWLEDGEMENTS}

The authors would like to thank Velia Hernández-García for her help with this manuscript.

\section{REFERENCES}

[1] Sainte-Rose C, Piatt JH, Renier D, et al. Mechanical complications in shunts. Pediatr Neurosurg 1992; 17(1): 2-9.

[2] Mizrachi IB, Trobe JD, Gebarski SS, Garton HJ. Papilledema in the assessment of ventriculomegaly. J Neuroophthalmol 2006; 26(4): 260-3.

[3] Arnell K, Eriksson E, Olsen L. Asymptomatic shunt malfunction detected fortuitously by observation of papilloedema. Acta Neurochir (Wien) 2003; 145(12): 1093-6.

[4] Nazir S, O'Brien M, Qureshi NH, Slape L, Green TJ, Phillips PH. Sensitivity of papilledema as a sign of shunt failure in children. J AAPOS 2009; 13(1): 63-6.

[5] Lee AG. Visual loss as the manifesting symptom of ventriculoperitoneal shunt malfunction. Am J Ophthalmol 1996; 122(1): 127-9.

[6] Katz DM, Trobe JD, Muraszko KM, Dauser RC. Shunt failure without ventriculomegaly proclaimed by ophthalmic findings. J Neurosurg 1994; 81(5): 721-5.

This is an open access article licensed under the terms of the Creative Commons Attribution Non-Commercial License (http://creativecommons.org/licenses/by-nc/3.0/) which permits unrestricted, non-commercial use, distribution and reproduction in any medium, provided the work is properly cited. 